\title{
Clinical utility of evolocumab in the management of hyperlipidemia: patient selection and follow-up
}

This article was published in the following Dove Press journal:

Drug Design, Development and Therapy

II July 2017

Number of times this article has been viewed

\author{
Dave L Dixon' \\ Leo F Buckley' \\ Cory R Trankle ${ }^{2}$ \\ Dinesh Kadariya ${ }^{3}$ \\ Antonio Abbate ${ }^{2}$ \\ 'Department of Pharmacotherapy \\ and Outcomes Science, School \\ of Pharmacy, ${ }^{2}$ Pauley Heart \\ Center, ${ }^{3}$ Department of Internal \\ Medicine, School of Medicine, \\ Virginia Commonwealth University, \\ Richmond,VA, USA
}

\begin{abstract}
Inhibition of PCSK9 is a novel therapeutic strategy aimed at reducing low-density-lipoprotein cholesterol (LDL-C) and cardiovascular risk. Evolocumab is a fully humanized monoclonal antibody that inhibits PCSK9, an enzyme that binds to LDL receptors and prevents them from recycling to the hepatocyte surface. Clinical trials have demonstrated $50 \%-70 \%$ reductions in LDL-C with evolocumab when used in combination with statin therapy. The recent FOURIER trial demonstrated that evolocumab further reduces cardiovascular events, but not mortality, in high-risk patients already receiving statin therapy. Furthermore, evolocumab did not affect neurocognitive function and was not associated with antidrugantibody production in over 60,000 patient-years of drug exposure. Appropriate candidates for evolocumab primarily are individuals at high cardiovascular risk, including those with familial hypercholesterolemia and/or established cardiovascular disease, who are already on statin therapy. At this time, the use of evolocumab monotherapy seems appropriate only for individuals deemed statin-intolerant despite attempting several statins. Consideration must be given toward patient willingness to self-inject evolocumab and issues concerning third-party coverage, given the current costs of evolocumab.
\end{abstract}

Keywords: evolocumab, PCSK9, familial hypercholesterolemia, hyperlipidemia

\section{Introduction}

A plethora of evidence has demonstrated the effectiveness of statins to significantly reduce low-density-lipoprotein cholesterol (LDL-C) and the risk of atherosclerotic cardiovascular disease (ASCVD). ${ }^{1}$ However, significant residual risk remains in statintreated patients and has led to the pursuit of other lipid-lowering therapies to use in combination with statins in hopes of reducing this residual risk. ${ }^{2}$ While ezetimibe has modest effects in reducing LDL-C and residual risk, ${ }^{3}$ the LDL-C hypothesis would suggest that more potent LDL-C-lowering therapies may have a greater impact on ASCVD-risk reduction.

PCSK9 is a novel target for lowering LDL-C. ${ }^{4}$ Synthesized in the liver, PCSK9 binds to the LDL receptor (LDL-R) resulting in endocytosis and lysosomal degradation (Figure 1). A clear genetic basis for targeting PCSK9 exists, as individuals with a gain-of-function mutation are at increased ASCVD risk, while those with a loss-offunction mutation appear to be at lower risk of ASCVD. ${ }^{5,6}$ As such, PCSK9 inhibition has become an attractive therapeutic target. In this review, we focus on evolocumab and the current evidence supporting its clinical use.

Department of Pharmacotherapy and

Outcomes Science, School of Pharmacy,

Virginia Commonwealth University,

410 North 12th Street, PO Box 980533 ,

Richmond, VA 23298-0533, USA

Tel +l 8046283784

Email dldixon@vcu.edu

\section{Pharmacology}

Evolocumab is a fully humanized monoclonal $\left(\mathrm{IgG}_{2}\right)$ antibody that fills a concave surface on the catalytic site of PCSK9, which is nearby the LDL-R-binding site. 


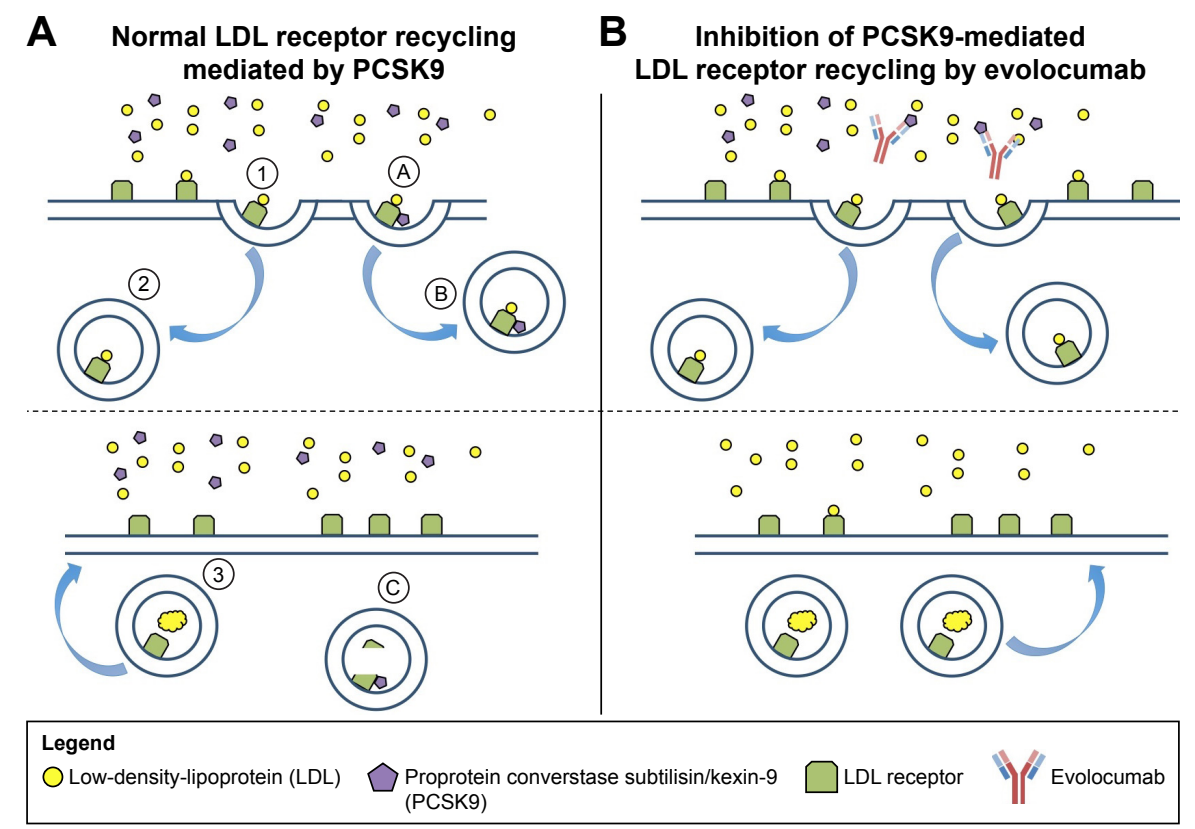

Figure I Inhibition of PCSK9 with evolocumab.

Notes: (A) The LDL receptor first binds circulating LDL (1). The LDL-LDL receptor complex is then internalized into the hepatocyte (2)), where LDL degradation occurs (3). After PCSK9 binds the LDL receptor (A)), the PCSK9-LDL receptor complex is internalized into the hepatocyte (B) and the LDL receptor metabolized (C)). (B) Binding of circulating PCSK9 by the anti-PCSK9 antibody evolocumab prevents the internalization of LDL receptors. Therefore, more LDL receptors are available on the surface of the hepatocyte for LDL clearance.

The binding of evolocumab to PCSK9 prevents PCSK9 from binding to the LDL-R, which prevents LDL-R degradation, allowing a return to the hepatocyte surface, where it can continue to remove LDL-C from the circulation. ${ }^{7}$

Evolocumab is administered via subcutaneous injection, and maximally suppresses circulating unbound PCSK9 within 4 hours. ${ }^{8}$ Peak serum concentrations with evolocumab are reached in 3-4 days, and bioavailability is estimated at $72 \%$. Evolocumab is eliminated mostly by saturation of the binding to PCSK9, while at higher concentrations elimination occurs through a nonsaturable proteolytic pathway. The half-life of evolocumab is approximately 11-17 days. Importantly, evolocumab is a large molecule at $144 \mathrm{kDa}$, suggesting a low likelihood of it crossing the blood-brain barrier and having any adverse effects on the central nervous system. ${ }^{8}$ Although evolocumab exposure decreases with increasing body weight, this effect is not thought to be clinically significant. Although no dose adjustment is required in patients with renal or hepatic impairment, individuals with severe renal or hepatic dysfunction have not been studied. Evolocumab does not have any significant drug-drug, drugfood, or drug-disease interactions.

\section{Efficacy studies}

Studies of evolocumab have demonstrated lipid-lowering efficacy across a broad patient population. In this section, we highlight the effects of evolocumab in patients with and without familial hypercholesterolemia (FH), statin intolerance, and common comorbidities, such as diabetes. In addition, we review evolocumab's effects on clinical outcomes, key intermediate and surrogate end points, and its safety profile. The lipoprotein-modifying effects of evolocumab in select studies are summarized in Table 1.

\section{Reduction of LDL-C General population}

As monotherapy, evolocumab $140 \mathrm{mg}$ biweekly and evolocumab $420 \mathrm{mg}$ once monthly lower LDL-C by approximately $47 \%-57 \%$ and $53 \%-55 \%$, respectively, after 12 weeks of treatment compared to placebo. ${ }^{12,13}$ Over 52 weeks of follow-up, repeated evolocumab dosing biweekly or once monthly maintains durable LDL-C lowering comparable to that shown in 12-week follow-up. ${ }^{11,14}$

Importantly, LDL-C lowering with evolocumab is maintained on statin background therapy. ${ }^{15}$ When evolocumab was added to background therapy with atorvastatin $80 \mathrm{mg}$ or atorvastatin $80 \mathrm{mg}$ plus ezetimibe, 12 weeks of evolocumab therapy reduced LDL-C by $76 \%$ and $47 \%$, respectively, for the $140 \mathrm{mg}$ biweekly regimen and by $71 \%$ and $39 \%$, respectively, for the $420 \mathrm{mg}$ once-monthly regimen. ${ }^{14}$ Robust LDL-C lowering with evolocumab as add-on therapy has 
Table I Effects of evolocumab on pro- and antiatherogenic lipoproteins

\begin{tabular}{|c|c|c|c|c|c|c|c|c|c|c|}
\hline Study & Population & Duration & Dosing & LDL-C & HDL-C & Non-HDL-C & TGs & ApoB & ApoAl & Lp(a) \\
\hline \multirow[t]{2}{*}{ Giugliano et al ${ }^{15}$ (LAPLACE) } & Non-FH & 12 weeks & $140 \mathrm{mg}$ Q2W & $-66.1 \%$ & $8.1 \%$ & $-61.4 \%$ & $-33.7 \%$ & $-56.4 \%$ & $0.3 \%$ & NR \\
\hline & & & $420 \mathrm{mg}$ Q4W & $-50.3 \%$ & $4.5 \%$ & $-47.6 \%$ & $-19.4 \%$ & $-42.0 \%$ & $3.6 \%$ & NR \\
\hline \multirow[t]{2}{*}{ Robinson et al ${ }^{16}$ (LAPLACE 2) } & Non-FH & 12 weeks & 140 mg Q2W & $-61.8 \%$ & $8.4 \%$ & $-54.4 \%$ & $-9.3 \%$ & $-49.1 \%$ & NR & $-25.0 \%$ \\
\hline & & & $420 \mathrm{mg}$ Q4W & $-65.1 \%$ & $7.8 \%$ & $-56.3 \%$ & $-15.4 \%$ & $-53.3 \%$ & NR & $-27.5 \%$ \\
\hline \multirow[t]{2}{*}{ Koren et al' ${ }^{12}$ (MENDEL) } & Non-FH & 12 weeks & $140 \mathrm{mg}$ Q2W & $-47.2 \%$ & $10.2 \%$ & $-45.2 \%$ & $-12 \%$ & $-44.2 \%$ & $10.5 \%$ & $-29.3 \%$ \\
\hline & & & $420 \mathrm{mg}$ Q4W & $-52.5 \%$ & $5.8 \%$ & $-47.1 \%$ & $-3.3 \%$ & $-42.5 \%$ & $5.3 \%$ & $-29.2 \%$ \\
\hline \multirow[t]{2}{*}{ Koren et $\mathrm{al}^{13}$ (MENDEL 2) } & Non-FH & 12 weeks & 140 mg Q2W & $-57.1 \%$ & $7.6 \%$ & $-49.8 \%$ & $-6.2 \%$ & $-47.8 \%$ & NR & $-20.4 \%$ \\
\hline & & & $420 \mathrm{mg}$ Q4W & $-54.8 \%$ & $5.5 \%$ & $-51.2 \%$ & $-17.7 \%$ & $-48.4 \%$ & NR & $-17.8 \%$ \\
\hline Blom et al'14 (DESCARTES) & Non-FH & 52 weeks & $420 \mathrm{mg}$ Q4W & $-59.3 \%$ & $5.4 \%$ & $-50.3 \%$ & $-11.5 \%$ & $-44.2 \%$ & $2.2 \%$ & $-22.4 \%$ \\
\hline Raal et $\mathrm{al}^{21}$ (RUTHERFORD) & $\mathrm{HeFH}$ & 12 weeks & $420 \mathrm{mg}$ Q4W & $-56.4 \%$ & $6.8 \%$ & $-53.5 \%$ & $-19.9 \%$ & $-46.2 \%$ & $1.7 \%$ & $-31.5 \%$ \\
\hline \multirow[t]{2}{*}{ Raal et $\mathrm{al}^{24}$ (RUTHERFORD 2) } & $\mathrm{HeFH}$ & 12 weeks & $140 \mathrm{mg}$ Q2W & $-59.2 \%$ & $9.2 \%$ & $-54.8 \%$ & $-19.6 \%$ & $-49.1 \%$ & $8.7 \%$ & $-31.6 \%$ \\
\hline & & & $420 \mathrm{mg}$ Q4W & $-61.3 \%$ & $9.1 \%$ & $-55.0 \%$ & $-11.6 \%$ & $-49.4 \%$ & $3.9 \%$ & $-28.2 \%$ \\
\hline $\begin{array}{l}\text { Stein et a } \mathrm{a}^{23} \\
(\text { TESLA A) }\end{array}$ & $\mathrm{HoFH}$ & 12 weeks & $420 \mathrm{mg}$ Q4W & $-16.5 \% *$ & $4.7 \% *$ & NR & $-5.7 \%$ & $-14.9 \% *$ & $1.3 \% *$ & $-24.6 \% *$ \\
\hline $\begin{array}{l}\text { Raal et a }{ }^{22} \\
\text { (TESLA B) }\end{array}$ & $\mathrm{HoFH}$ & 12 weeks & $420 \mathrm{mg}$ Q4W & $-30.9 \%$ & $-0.1 \%$ & NR & $0.3 \%$ & $-23.1 \%$ & $N R$ & $-11.8 \%$ \\
\hline
\end{tabular}

Notes: *Percentage change from baseline (without placebo correction). Percentage changes in lipoprotein subfractions are summarized for evolocumab. Only results for US Food and Drug and Drug Administration-approved regimens are included.

Abbreviations: LDL-C, low-density-lipoprotein cholesterol; HDL-C, high-density-lipoprotein cholesterol; TGs, triglycerides; FH, familial hypercholesterolemia; HeFH, heterozygous $\mathrm{FH}$; HoFH, homozygous FH; NR, not reported; Q2W, every two weeks.

been shown across several statins and statin doses, with or without ezetimibe. ${ }^{17}$

When added to background ezetimibe without statin therapy, PCSK9 inhibition with evolocumab reduces LDL-C by an additional 34\%-39\%. ${ }^{12,13}$ Evolocumab has also demonstrated similar LDL-C lowering effects in Japanese patients, suggesting that the utility of monoclonal antibodies against PCSK9 may not be modified by race or ethnicity. ${ }^{17,18}$ Additional analyses in other races/ethnicities would be of interest.

In patients without $\mathrm{FH}$, the extremely low levels of LDL-C achieved by adding evolocumab to high-intensity statin therapy are unprecedented. For example, in the Phase II LAPLACE 2 study, patients receiving rosuvastatin $40 \mathrm{mg}$ daily and evolocumab $420 \mathrm{mg}$ monthly achieved a mean LDL-C of $33 \mathrm{mg} / \mathrm{dL}(\mathrm{SD} 28.3 \mathrm{mg} / \mathrm{dL}) .{ }^{19} \mathrm{In}$ the long-term DESCARTES study, $41 \%$ and $66 \%$ of patients achieved at least one LDL-C $<15 \mathrm{mg} / \mathrm{dL}$ and $<25 \mathrm{mg} / \mathrm{dL}$, respectively. ${ }^{20}$

\section{FH}

Evolocumab has also demonstrated meaningful lipidlowering efficacy in patients with $\mathrm{FH}$ when added to standard-of-care lipid-lowering agents. In homozygous FH, evolocumab $420 \mathrm{mg}$ monthly reduced LDL-C by $41 \%$ (95\% CI $28 \%-53 \%$ ) for patients with one or more defective LDL-R alleles compared to placebo. ${ }^{21}$ LDL-C reductions were less pronounced when the analysis was restricted to homozygous patients with defective/negative LDL-R alleles $(25 \%, 95 \%$
CI 7\%-42\%), ${ }^{22}$ and were not different than placebo in doublenegative LDL-R allele patients. ${ }^{23}$

In heterozygous $\mathrm{FH}$, evolocumab $140 \mathrm{mg}$ biweekly and $420 \mathrm{mg}$ monthly reduced LDL-C by $59 \%$ (95\% CI $53 \%-65 \%)$ and $61 \%$ (95\% CI 54\%-69\%), respectively. ${ }^{24,25}$ Whereas LDL-C reductions are influenced by genotype in homozygous FH, LDL-C reductions are not different across differences in genotype among patients with heterozygous $\mathrm{FH} .^{24}$

LDL apheresis removes $52 \%$ of circulating PCSK9, suggesting that evolocumab's LDL-C-lowering effects may be blunted when utilized in conjunction with LDL apheresis. ${ }^{26}$ However, a post hoc analysis of a clinical trial of homozygous FH found similar mean LDL-C reductions at weeks 12 and 48 between subjects, regardless of whether or not they were on apheresis. ${ }^{27}$

\section{Effects beyond LDL cholesterol Apolipoprotein B}

In addition to LDL-C reductions, evolocumab has demonstrated reductions in ApoB that mirror LDL-C changes. Compared to placebo, ApoB was reduced by $45 \%-48 \%$ when evolocumab was added to background statin therapy of any intensity for 1 year of treatment. ${ }^{28}$

Adding evolocumab to ezetimibe-containing statin regimens produced a slightly less significant drop in ApoB. In a 12-week study, evolocumab reduced ApoB by $34 \%-38 \%$ when added to statin therapy with ezetimibe compared to $47 \%-61 \%$ when added to statin therapy without ezetimibe. ${ }^{17}$ 


\section{High-density lipoprotein and apolipoprotein AI}

Increases in nonatherogenic lipoprotein subsets are modest with evolocumab treatment. When added to atorvastatin $10 \mathrm{mg}$, atorvastatin $80 \mathrm{mg}$, or atorvastatin $80 \mathrm{mg}$ plus ezetimibe, placebo-adjusted changes in highdensity-lipoprotein (HDL) were 5.4\% (SE 1.1\%) after 52 weeks of treatment. ${ }^{28}$ Even smaller changes were observed in evolocumab-treated patients with respect to ApoA1 $(<5 \%$ on average). ${ }^{28}$

\section{Lipoprotein(a)}

Lipoprotein(a) (Lp[a]) consists of ApoA, a plasminogen-like protein that may interfere with fibrinolysis covalently bound to the ApoB of an LDL particle, and has been associated with cardiovascular risk. ${ }^{29}$ Data from clinical trials of evolocumab monotherapy (MENDEL and MENDEL 2), evolocumab add-on therapy (LAPLACE and LAPLACE 2), evolocumab in heterozygous FH (RUTHERFORD and RUTHERFORD 2) and long-term extension trials (OSLER and OSLER 2) were pooled to determine the effects of evolocumab on circulating Lp(a) levels. ${ }^{30}$

After 12 weeks of treatment, evolocumab-treated patients experienced mean reductions in Lp(a) of $25 \%$ (95\% CI $4 \%-40 \%)$ and $22 \%(95 \%$ CI $4 \%-40 \%)$ for the $140 \mathrm{mg}$ biweekly and the $420 \mathrm{mg}$ monthly regimens, respectively. $\mathrm{Lp}$ (a) reductions were durable over 52 weeks of treatment in long-term extension trials and related to LDL-C reductions. ${ }^{11}$ Evolocumab-induced reductions in $\mathrm{Lp}(\mathrm{a})$ appear unaffected by age, sex, race/ethnicity, diabetes status, or intensity of statin therapy. ${ }^{17,31}$ The recent testing of an antisense oligonucleotide against ApoA in humans may herald a future increase in our understanding of $\mathrm{Lp}(\mathrm{a}){ }^{32}$

\section{Special populations Metabolic disease}

The effects of evolocumab have been investigated specifically in patients with type 2 diabetes, who are at heightened cardiovascular risk. ${ }^{33} \mathrm{~A}$ comparison of patients with and without type 2 diabetes mellitus enrolled in Phase II evolocumab clinical trials revealed no significant differences in changes in LDL-C, Lp(a), or HDL. ${ }^{34}$ However, in a separate analysis of the DESCARTES study, LDL-C lowering was significantly greater in patients without type 2 diabetes or with impaired fasting glucose or metabolic syndrome compared to those with type 2 diabetes. ${ }^{35}$ Notably, the former analysis included a larger sample size $(n=2,531$ vs $n=901)$ and multiple studies.

\section{Statin intolerance}

After rechallenging patients with atorvastatin to verify statin intolerance, Nissen et al randomized patients to receive ezetimibe $10 \mathrm{mg}$ daily or evolocumab $420 \mathrm{mg}$ once monthly. ${ }^{36}$ LDL-C was decreased by $17 \%$ (95\% CI 13\%-21\%) and 53\% $(50 \%-56 \%)$ in ezetimibe- and evolocumab-treated patients, respectively $(P<0.001$ for comparison of ezetimibe and evolocumab). Similar effects were seen for evolocumab $140 \mathrm{mg}$ every 2 weeks in comparison with placebo. ${ }^{37}$ When added to ezetimibe background therapy in statin-intolerant patients, evolocumab $420 \mathrm{mg}$ once monthly reduced LDL-C by $47 \%(95 \%$ CI $41 \%-54 \%){ }^{38}$

\section{Clinical outcomes FOURIER trial}

In March 2017, the FOURIER trial was published. ${ }^{9}$ This outcomes trial randomized 27,564 patients with ASCVD and LDL-C $>70 \mathrm{mg} / \mathrm{dL}$ to receive evolocumab or placebo in addition to background statin therapy. Nearly $70 \%$ of patients were receiving high-intensity statins, and the median LDL-C was $92 \mathrm{mg} / \mathrm{dL}$ (IQR 80-109). The primary efficacy end point was the composite of cardiovascular death, myocardial infarction, stroke, hospitalization for unstable angina, or coronary revascularization.

After 48 weeks of treatment, patients in the evolocumab arm had a median LDL-C of $30 \mathrm{mg} / \mathrm{dL}$ (IQR 19-46). An LDL-C of $<25 \mathrm{mg} / \mathrm{dL}$ was achieved in $42 \%$ of evolocumab patients compared to $0.1 \%$ of placebo patients. The median duration of follow-up in this event-driven trial was 26 months (IQR 22-30).

Overall, when added to background statin therapy, evolocumab significantly reduced the risk of the primary end point compared to placebo ( $9.8 \%$ vs $11.3 \%$, HR $0.85,95 \%$ CI $0.79-0.92 ; P<0.001)$. The between-group difference in the primary end point was due to significant reductions in myocardial infarction (3.4\% vs $4.6 \%$, HR $0.73,95 \%$ CI $0.65-0.82 ; P<0.001$ ), stroke ( $1.5 \%$ vs $1.9 \%$, HR $0.79 ; 95 \%$ CI $0.66-0.95 ; P<0.01)$, and coronary revascularization (5.5\% vs $7.0 \%$, HR $0.78,95 \%$ CI $0.71-0.86$; $P<0.001)$. There were no significant differences in cardiovascular death, death from any cause, or hospitalization for unstable angina. Event reductions were similar across subgroups.

Importantly, adverse events leading to drug discontinuation were not significantly different between evolocumab and placebo (1.6\% vs $1.5 \%)$. Injection-site reactions were slightly more common in the evolocumab group $(2.1 \%$ vs $1.6 \%$ ), but occurred infrequently overall. The risks of neurocognitive events and new-onset diabetes were 
not significantly different between the two groups. Over 60,000 patient-years of evolocumab exposure, there were only three patients with antidrug antibodies, none of which was neutralizing antibodies.

Overall, the FOURIER trial supports the LDL-C hypothesis of ASCVD and demonstrates that extreme LDL-C reduction prevents ASCVD when added to background statin therapy. Notably, the median duration of FOURIER was approximately 3 years less than the duration of landmark statin trials, and a mortality benefit was not observed during the trial. The investigators conducted a landmark analysis of FOURIER years $0-1$ and years $1-2$. In comparison with a lipid lowering meta-analysis, ${ }^{1}$ FOURIER event reductions were similar to those reported in years $0-1$ and years $1-2$ of prior lipid-lowering trials. This important comparison suggests that longer follow-up of FOURIER patients would reveal a significant mortality reduction. Indeed, evolocumab may require 1-2 years before changes in coronary-artery atheroma volume can be detected. ${ }^{10}$

In an exploratory analysis of the OSLER and OSLER 2 clinical trials, evolocumab demonstrated a 53\% relative reduction in the composite of cardiovascular death, myocardial infarction, unstable angina requiring hospitalization, coronary revascularization, stroke, transient ischemic attack, and hospitalization for heart failure after 1 year of treatment. ${ }^{11}$ Differences in effect size between FOURIER and OSLER may be attributed to the inclusion of very high-risk patients with FH, smaller samples, and shorter duration of follow-up in OSLER, which may have predisposed to artificial magnification of treatment effect.

\section{Safety of evolocumab Short-term adverse-effect profile}

PCSK9 inhibition with evolocumab is well tolerated in the short term. The most common adverse effects are nasopharyngitis (10.5\% vs $9.6 \%$ for placebo), upper respiratory tract infection $(9.3 \%$ vs $6.3 \%)$, influenza $(7.5 \%$ vs $6.3 \%$ for placebo), back pain (6.2\% vs $5.6 \%$ for placebo), and injection-site reactions, such as erythema, pain, and bruising (5.7\% vs $5 \%$ for placebo).

\section{Long-term adverse-effect profile}

The long-term adverse-effect profile of evolocumab is less clear, due to a lack of long-term follow-up on this new agent. It has been hypothesized that exposure to extremely low LDL-C levels would result in pathologic cholesterol deficiency, impairing cell-membrane integrity and cholesteroldependent functions, such as steroid-hormone production. ${ }^{39}$
Moreover, PCSK9 is expressed in the central nervous system and appears to exert protective effects, ${ }^{40}$ further intensifying neurocognitive concerns.

On the other hand, elderly patients with PCSK9 loss-offunction mutations demonstrate similar cognitive function as those with wild-type PCSK 9 genotypes, ${ }^{41}$ and non-LDL-C pathways (eg, dietary absorption, hepatic de novo synthesis) appear sufficient to maintain adequate cholesterol availability in the setting of abnormally low LDL levels. ${ }^{42}$ Long-term follow-up of patients utilizing PCSK9 inhibitors will be required to resolve this controversy.

\section{Neurocognitive effects}

Khan et al conducted a meta-analysis of eleven PCSK9inhibitor studies (evolocumab and alirocumab), involving 10,656 patients. ${ }^{43}$ Overall, the rate of serious adverse events was not significantly different between PCSK9 inhibitortreated patients and controls (11\% vs $10.3 \%$, odds ratio 1 ; $P=0.96)$. Neurocognitive events, which can be either acute (eg, confusion) or chronic (eg, dementia), did not occur more frequently in PCSK9 inhibitor-treated patients, and overall event rates were low $(0.8 \%$ for PCSK 9 inhibitors vs $0.5 \%$ for control; OR 1.29, 95\% CI 0.64-2.59; $P=0.47)$. Excluding short-term studies, the incidence of any neurocognitive event was significantly increased in PCSK9-inhibitor patients (OR 2.81, 95\% CI 1.32-5.99; $P=0.007$ ).

In the EBBINGHAUS study, 1,974 patients were recruited from the overall FOURIER cohort and followed prospectively for the duration of the FOURIER trial. ${ }^{44}$ The Cambridge Neuropsychological Test automated battery was administered to assess neurocognitive function. Overall, there were no significant differences in the primary or secondary neurocognitive function end points between evolocumabtreated and placebo-treated patients $(P<0.0001$ for noninferiority for the primary end point). There were no differences either in cognitive tests when subjects were stratified by their achieved LDL-C, including $<25 \mathrm{mg} / \mathrm{dL}$. Furthermore, no adverse neurocognitive effects were found in alirocumabtreated patients with LDL-C $<25 \mathrm{mg} / \mathrm{dL}$ compared to alirocumab-treated patients with LDL-C $\geq 25 \mathrm{mg} / \mathrm{dL}$ over a median exposure to extremely low LDL-C of 43 weeks. ${ }^{19}$ Despite these data, additional studies are warranted to investigate further the neurocognitive effects of longer term PCSK9-inhibitor use.

\section{Cholesterol synthesis and absorption}

Vitamin E and steroid-hormone levels were measured in 100 patients who participated in a substudy of a long-term 
evolocumab study. ${ }^{20}$ Relative to changes in cholesterol, there were no significant changes in serum vitamin E levels, LDL vitamin E levels, or red-cell membrane vitamin E levels. Similarly, estrogen, testosterone, cortisol, and adrenocorticotropic hormone were unaffected by extreme LDL lowering with evolocumab.

In an 88-patient substudy of the Phase II MENDEL study, changes in cholesterol-synthesis markers (lathosterol and desmosterol) and in cholesterol-absorption markers (campesterol and $\beta$-sitosterol) were evaluated after 12 weeks of treatment with evolocumab or ezetimibe. ${ }^{45}$ Both evolocumab $140 \mathrm{mg}$ biweekly and $420 \mathrm{mg}$ monthly reduced absolute levels of all four cholesterols.

However, after adjustment for changes in total cholesterol, the net effects appeared neutral. Evolocumab $140 \mathrm{mg}$ biweekly and $420 \mathrm{mg}$ monthly regimens increased the $\beta$-sitosterol:total cholesterol (TC) ratio $(P<0.05$ for both regimens) and the campesterol:TC ratio (not significant for either regimen), suggesting a compensatory increase in cholesterol absorption.

Evolocumab $420 \mathrm{mg}$ monthly increased desmosterol:TC $(P<0.01)$ but not lathosterol:TC ratio (not significant), and thus had unclear effects on cholesterol synthesis. Evolocumab $140 \mathrm{mg}$ biweekly had no significant effect on either campesterol:TC ratio or lathosterol:TC ratio. Given the small sample of this study, additional data are required for a more definitive analysis of the effects of evolocumab on cholesterol synthesis and absorption.

\section{Patient selection}

The 2013 American College of Cardiology/American Heart Association blood cholesterol guideline makes no mention of PCSK9 inhibitors, as these agents were not approved when the guidelines were published. ${ }^{46}$ In 2016 , an expert-consensus document was published to inform clinicians regarding the use of non-statins, including PCSK9 inhibitors. ${ }^{47}$ These recommendations were developed based on the 2013 guidelines and include algorithms describing workflows for considerations regarding the use of nonstatins. Essentially, PCSK9 inhibitors were recommended for secondary-prevention patients (with or without diabetes) needing additional LDL-C lowering and those with LDL-C $\geq 90 \mathrm{mg} / \mathrm{dL}$ (ie, FH). Notably, the FOURIER trial demonstrated that evolocumab reduces cardiovascular events in these high-risk patients when used in combination with statin therapy. ${ }^{11}$

Also in 2016, the European Society of Cardiology and European Atherosclerosis Society published a consensus statement on the use of PCSK9 inhibitors. ${ }^{48}$ Essentially,
PCSK9 inhibitors were recommended in individuals with documented ASCVD, severe FH without ASCVD, and those with diabetes and evidence of target organ damage (eg, proteinuria) or a major risk factor (eg, hypertension). Furthermore, PCSK9 inhibitors are recommended in these very high-risk patients only if their LDL-C remains elevated after titration to a maximally tolerated statin dose plus ezetimibe. Additional guidance is given regarding the identification of statin-associated myalgia and its management to encourage clinicians to ensure very high-risk patients are receiving the maximally tolerated dose of a statin.

Patients who require lipid-lowering therapy but have confirmed intolerance to statin therapy would also be appropriate candidates for evolocumab. Statin intolerance is generally defined as the inability to tolerate at least two statins, including one at the lowest starting dose and another at any dose. ${ }^{49}$ Additionally, statin intolerance should be confirmed by using either laboratory data (eg, creatine kinase) or improvement in symptoms after decreasing the dose or discontinuing the statin. The achievement of goal lipid levels in high-risk patients is challenging without the ability to increase or utilize moderate- to high-dose statin therapy. The GAUSS 3 trial $^{36}$ enrolled patients with prior statin intolerance due to muscle-related events (myalgia, myositis, or rhabdomyolysis), confirming their statin intolerance with a placebo-controlled atorvastatin rechallenge. Once true statin intolerance was confirmed, evolocumab therapy showed superior LDL-C reduction and a comparably low rate of further muscle-related events when compared to ezetimibe, strengthening its emerging role as a statin alternative for this particular group.

The use of evolocumab for primary prevention is more controversial. While using evolocumab in patients with FH who have not yet had a cardiovascular event would be appropriate if additional LDL-C lowering was needed, statins should remain the initial drug of choice for primary prevention. Ezetimibe and bile-acid sequestrants should be added to statin therapy if additional LDL-C lowering is needed instead of evolocumab. ${ }^{47}$ The role of evolocumab in the general primary-prevention population warrants further study, and its cost-effectiveness in this population remains unclear.

\section{Patient perspectives}

The cost of both evolocumab and alirocumab, which have a mean annual wholesale price of US\$14,350, is a major barrier for patient access and the crux of ongoing discussions on who will pay for these drugs and which patients 
should receive these agents. Kazi et $\mathrm{al}^{50}$ performed a costeffectiveness analysis of PCSK9 inhibitors in patients with heterozygous FH or CVD using the Cardiovascular Disease Policy Model, which is a simulation model of US adults aged 35-94 years. An annual wholesale cost of $\$ 14,350$ was used in the analysis, which was based on the average cost for both alirocumab and evolocumab. Using a qualityadjusted life-year cost-effectiveness threshold of $\$ 100,000$, the study found that the annual cost of PCSK9 inhibitors would need to be reduced nearly $70 \%$ to $\$ 4,356$. The total drug costs over 5 years would approach $\$ 600$ billion if all eligible patients received a PCSK9 inhibitor. It is important to note that this analysis was based on a simulation model and before the clinical outcome benefit of PCSK9 inhibitors was established. Additionally, actual drug costs are usually lower, as final pricing is negotiated between pharmaceutical companies and payers.

Unsurprisingly, coverage of PCSK9 inhibitors by thirdparty payers in the US has been poor. Initial rejection rates have been reportedly as high as $80 \%$, with only $25 \%-50 \%$ getting approved after additional attempts. ${ }^{51}$ While this may be expected for PCSK9-inhibitor requests in the primaryprevention population and those without $\mathrm{FH}$, rejection rates in patients with $\mathrm{FH}$ have also been reported to be as high as $80 \%{ }^{51}$ The prior-authorization process can place significant strain on medical practices and health systems, especially if multiple attempts are required to obtain approval. As for other countries, especially those in the EU, coverage of PCSK9 inhibitors varies as well, but there are fewer barriers, such as the lack of a complicated prior-authorization process due to having a single-payer health-care system. ${ }^{48}$ It is unknown at this time how the FOURIER trial results will influence drug pricing and the willingness of third-party payers to provide adequate coverage for PCSK9 inhibitors.

In addition to the high costs of PCSK9 inhibitors, the only currently available drug-delivery option for both evolocumab and alirocumab is subcutaneous injection. In an investigation of alirocumab-delivery devices (syringe or prefilled autoinjector pen), which are essentially the same as evolocumab's, primary-care providers and specialist providers provided favorable feedback on the ease of use of the autoinjector pen and enthusiasm that many or most of their patients would be willing to use the delivery devices, particularly the pen. Patients similarly found the delivery modes easy to use and understand, and after instructions $72 \%$ of them were "very willing" to self-inject with the pen. ${ }^{52}$ Of note, having already self-injected investigational drugs as part of their participation in the OSLER parent trials, $74.1 \%$ of the eligible patients were enrolled in subsequent OSLER and OSLER 2 trials. ${ }^{11}$ The reasons for eligible patients not enrolling was not reported, but this reflects a sizeable majority of subjects who demonstrated voluntary adherence.

Evolocumab can be administered once monthly by either injecting three doses within 30 minutes from prefilled autoinjector cartridges or by a single 9-minute administration from an on-body infuser. The latter device even allows for moderate physical activities during the injection process. ${ }^{8}$ The THOMAS and THOMAS 2 trials demonstrated that after appropriate training, self-administration of evolocumab at home is safe and effective, whether a prefilled syringe, an autoinjector, or an automated minidoser is used. ${ }^{53}$ Interestingly, when patients enrolled in the FOURIER trial were given the option of $150 \mathrm{mg}$ every two weeks or $450 \mathrm{mg}$ every four weeks, only about $10 \%$ appeared to have received the latter dosing, although all doses in this trial were given via syringe, and investigational product availability may have a limited 4-week dosing option. ${ }^{9}$ Recently, the US Food and Drug and Drug Administration also approved a once-monthly option for alirocumab, which involves administering two $150 \mathrm{mg}$ injections for a total dose of $300 \mathrm{mg}$. It should also be noted that oral formulations of PCSK9 inhibitors are in development. ${ }^{4}$

In addition to low rates of muscle-related events, evolocumab's side-effect profile appears extremely favorable. The FOURIER trial demonstrated no significant difference between evolocumab and placebo with regard to musclerelated events, cataracts, neurocognitive adverse events, and hemorrhagic stroke. ${ }^{9}$ Injection-site reactions occurred in $2.1 \%$ of patients receiving evolocumab compared to $1.6 \%$ receiving placebo; only $0.1 \%$ in each group discontinued therapy with the investigational injections due to these reactions. Overall adverse events leading to discontinuation of the investigational drug were not statistically different between groups: $1.6 \%$ with evolocumab and $1.5 \%$ with placebo. ${ }^{9}$ Considering the concerns many patients have regarding statin-associated side effects, whether real or perceived, the side-effect profile of evolocumab may be preferred by many patients.

\section{Conclusion}

PCSK9 has proven to be a highly viable therapeutic target. Evolocumab is a monoclonal antibody shown to be safe and effective in significantly reducing LDL-C. Recent evidence from the FOURIER trial has also demonstrated that evolocumab improves clinical outcomes. While evolocumab seems most appropriate for patients with $\mathrm{FH}$ or those with 
established CVD in need of additional LDL-C lowering, it is also an appealing option for statin-intolerant individuals who are at high risk. Limitations to using evolocumab include subcutaneous administration, patient-access issues, and unfavorable cost-effectiveness based on its current average wholesale price.

\section{Disclosure}

AA has received research funding from Regeneron and Sanofi. The other authors report no conflicts of interest in this work.

\section{References}

1. Cholesterol Treatment Trialists' (CTT) Collaborators. The effects of lowering LDL cholesterol with statin therapy in people at low risk of vascular disease: meta-analysis of individual data from 27 randomised trials. Lancet. 2012;380:581-590.

2. Reith C, Armitage J. Management of residual risk after statin therapy. Atherosclerosis. 2016;245:161-170.

3. Cannon CP, Blazing MA, Giugliano RP, et al. Ezetimibe added to statin therapy after acute coronary syndromes. $N$ Engl J Med. 2015;372: 2387-2397.

4. Dixon DL, Trankle C, Buckley L, et al. A review of PCSK9 inhibition and its effects beyond LDL receptors. J Clin Lipidol. 2016;10: 1073-1080.

5. Cohen JC, Boerwinkle E, Mosley TH, Hobbs HH. Sequence variations in PCSK9, low LDL, and protection against coronary heart disease. N Engl J Med. 2006;354:1264-1272.

6. Ference BA, Robinson JG, Brook RD, et al. Variation in PCSK9 and HMGCR and risk of cardiovascular disease and diabetes. NEngl J Med. 2016;375:2144-2153.

7. Page MM, Watts GF. Evolocumab in the treatment of dyslipidemia: preclinical and clinical pharmacology. Expert Opin Drug Metab Toxicol. 2015;11:1505-1515.

8. Repatha [package insert]. Thousand Oaks (CA): Amgen Inc; 2016.

9. Sabatine MS, Giugliano RP, Keech AC, et al. Evolocumab and clinical outcomes in patients with cardiovascular disease. N Engl J Med. 2017; 376:1713-1722.

10. Nicholls SJ, Puri R, Anderson T, et al. Effect of evolocumab on progression of coronary disease in statin-treated patients: the GLAGOV randomized clinical trial. JAMA. 2016;316:2373-2384.

11. Sabatine MS, Giugliano RP, Wiviott SD, et al. Efficacy and safety of evolocumab in reducing lipids and cardiovascular events. $N$ Engl $J$ Med. 2015;372:1500-1509.

12. Koren MJ, Scott R, Kim JB, et al. Efficacy, safety, and tolerability of a monoclonal antibody to proprotein convertase subtilisin/kexin type 9 as monotherapy in patients with hypercholesterolaemia (MENDEL): a randomised, double-blind, placebo-controlled, phase 2 study. Lancet. 2012;380:1995-2006.

13. Koren MJ, Lundqvist P, Bolognese M, et al. Anti-PCSK9 monotherapy for hypercholesterolemia: the MENDEL-2 randomized, controlled phase III clinical trial of evolocumab. J Am Coll Cardiol. 2014;63: 2531-2540.

14. Blom DJ, Hala T, Bolognese M, et al. A 52-week placebo-controlled trial of evolocumab in hyperlipidemia. N Engl J Med. 2014;370: 1809-1819.

15. Giugliano R, Desai N, Kohli P, et al. Efficacy, safety, and tolerability of a monoclonal antibody to proprotein convertase subtilisin/kexin type 9 in combination with a statin in patients with hypercholesterolaemia (LAPLACE-TIMI 57): a randomised, placebo-controlled, dose-ranging, phase 2 study. Lancet. 2012;380:2007-2017.
16. Robinson JG, Nedergaard BS, Rogers WJ, et al. Effect of evolocumab or ezetimibe added to moderate- or high-intensity statin therapy on LDL-C lowering in patients with hypercholesterolemia: the LAPLACE-2 randomized clinical trial. JAMA. 2014;311:1870-1882.

17. Hirayama A, Honarpour N, Yoshida M, et al. Effects of evolocumab (AMG 145), a monoclonal antibody to PCSK9, in hypercholesterolemic, statin-treated Japanese patients at high cardiovascular risk: primary results from the phase 2 YUKAWA study. Circ J. 2014;78:1073-1082.

18. Kiyosue A, Honarpour N, Kurtz C, Xue A, Wasserman SM, Hirayama A. A phase 3 study of evolocumab (AMG 145) in statin-treated Japanese patients at high cardiovascular risk. Am J Cardiol. 2016;117:40-47.

19. Robinson JG, Rosenson RS, Farnier M, et al. Safety of very low lowdensity lipoprotein cholesterol levels with alirocumab: pooled data from randomized trials. J Am Coll Cardiol. 2017;69:471-482.

20. Blom DJ, Djedjos CS, Monsalvo ML, et al. Effects of evolocumab on vitamin E and steroid hormone levels: results from the 52-week, phase 3, double-blind, randomized, placebo-controlled DESCARTES study. Circ Res. 2015;117:731-741.

21. Raal F, Scott R, Somaratne R, et al. Low-density lipoprotein cholesterollowering effects of AMG 145, a monoclonal antibody to proprotein convertase subtilisin/kexin type 9 serine protease in patients with heterozygous familial hypercholesterolemia: the reduction of LDL-C with PCSK9 inhibition in heterozygous familial hypercholesterolemia disorder (RUTHERFORD) randomized trial. Circulation. 2012;126: 2408-2417.

22. Raal FJ, Honarpour N, Blom DJ, et al. Inhibition of PCSK9 with evolocumab in homozygous familial hypercholesterolaemia (TESLA part B): a randomised, double-blind, placebo-controlled trial. Lancet. 2015;385:341-350.

23. Stein E, Honarpour N, Wasserman S, Xu F, Scott R, Raal F. Effect of the proprotein convertase subtilisin/kexin 9 monoclonal antibody, AMG 145, in homozygous familial hypercholesterolemia. Circulation. 2013;128:2113-2120.

24. Raal FJ, Stein EA, Dufour R, et al. PCSK9 inhibition with evolocumab (AMG 145) in heterozygous familial hypercholesterolaemia (RUTHERFORD-2): a randomised, double-blind, placebo-controlled trial. Lancet. 2015;385:331-340.

25. Raal F, Scott R, Somaratne R, et al. Low-density lipoprotein cholesterollowering effects of AMG 145, a monoclonal antibody to proprotein convertase subtilisin/kexin type 9 serine protease in patients with heterozygous familial hypercholesterolemia. Circulation. 2012;126: $2408-2417$.

26. Tavori H, Giunzioni I, Linton MF, Fazio S. Loss of plasma proprotein convertase subtilisin/kexin 9 (PCSK9) after lipoprotein apheresis. Circ Res. 2013;113:1290-1295.

27. Raal FJ, Hovingh GK, Blom D, et al. Long-term treatment with evolocumab added to conventional drug therapy, with or without apheresis, in patients with homozygous familial hypercholesterolaemia: an interim subset analysis of the open-label TAUSSIG study. Lancet Diabetes Endocrinol. 2017;5:280-290.

28. Blom DJ, Hala T, Bolognese M, et al. A 52-week placebo-controlled trial of evolocumab in hyperlipidemia. $N$ Engl J Med. 2014;370: 1809-1819.

29. Clarke R, Peden JF, Hopewell JC, et al. Genetic variants associated with Lp(a) lipoprotein level and coronary disease. N Engl J Med. 2009; 361:2518-2528.

30. Raal FJ, Giugliano RP, Sabatine MS, et al. PCSK9 inhibition-mediated reduction in $\mathrm{Lp}$ (a) with evolocumab: an analysis of 10 clinical trials and the LDL receptor's role. J Lipid Res. 2016;57:1086-1096.

31. Desai N, Kohli P, Giugliano R, et al. AMG145, a monoclonal antibody against proprotein convertase subtilisin kexin type 9 , significantly reduces lipoprotein(a) in hypercholesterolemic patients receiving statin therapy. Circulation. 2013;128:962-969.

32. Viney NJ, van Capelleveen JC, Geary RS, et al. Antisense oligonucleotides targeting apolipoprotein(a) in people with raised lipoprotein(a): two randomised, double-blind, placebo-controlled, dose-ranging trials. Lancet. 2016;388:2239-2253. 
33. Shah AD, Langenberg C, Rapsomaniki E, et al. Type 2 diabetes and incidence of cardiovascular diseases: a cohort study in 1.9 million people. Lancet Diabetes Endocrinol. 2015;3:105-113.

34. Sattar N, Preiss D, Robinson JG, et al. Lipid-lowering efficacy of the PCSK9 inhibitor evolocumab (AMG 145) in patients with type 2 diabetes: a meta-analysis of individual patient data. Lancet Diabetes Endocrinol. 2016;4:403-410.

35. Blom DJ, Koren MJ, Roth E, et al. Evaluation of the efficacy, safety and glycaemic effects of evolocumab (AMG 145) in hypercholesterolaemic patients stratified by glycaemic status and metabolic syndrome. Diabetes Obes Metab. 2017;19:98-107.

36. Nissen SE, Stroes E, Dent-Acosta RE, et al. Efficacy and tolerability of evolocumab vs ezetimibe in patients with muscle-related statin intolerance. JAMA. 2016;315:1580-1590.

37. Stroes E, Colquhoun D, Sullivan D, et al. Anti-PCSK9 antibody effectively lowers cholesterol in patients with statin intolerance: the GAUSS-2 randomized, placebo-controlled phase 3 clinical trial of evolocumab. J Am Coll Cardiol. 2014;63:2541-2548.

38. Sullivan D, Olsson AG, Scott R, et al. Effect of a monoclonal antibody to PCSK9 on low-density lipoprotein cholesterol levels in statin-intolerant patients. JAMA. 2012;308:2497-2506.

39. Swiger KJ, Martin SS. PCSK 9 inhibitors and neurocognitive adverse events: exploring the FDA directive and a proposal for n-of-1 trials. Drug Saf. 2015;38:519-526.

40. Schulz R, Schlüter KD, Laufs U. Molecular and cellular function of the proprotein convertase subtilisin/kexin type 9 (PCSK9). Basic Res Cardiol. 2015;110:4

41. Postmus I, Trompet S, de Craen AJ, et al. PCSK9 SNP rs11591147 is associated with low cholesterol levels but not with cognitive performance or noncardiovascular clinical events in an elderly population. J Lipid Res. 2013;54:561-566.

42. Qamar A, Bhatt DL. Effect of low cholesterol on steroid hormones and vitamin E levels. Circ Res. 2015;117:662-664.

43. Khan AR, Bavishi C, Riaz H, et al. Increased risk of adverse neurocognitive outcomes with proprotein convertase subtilisinkexin type 9 inhibitors. Circ Cardiovasc Qual Outcomes. 2017; 10:e003153.
44. Koren MJ, Sabatine MS, Giugliana RP, et al. Long-term low-density lipoprotein cholesterol-lowering efficacy, persistence, and safety of evolocumab in treatment of hypercholesterolemia. JAMA Cardiol. 2017;2:598-607.

45. Peach M, Xu R, Fitzpatrick D, et al. Effect of evolocumab on cholesterol synthesis and absorption. J Lipid Res. 2016;57:2217-2224.

46. Stone NJ, Robinson J, Lichtenstein A, et al. 2013 ACC/AHA guideline on the treatment of blood cholesterol to reduce atherosclerotic cardiovascular risk in adults: a report of the American College of Cardiology/ American Heart Association Task Force on Practice Guidelines. J Am Coll Cardiol. 2014;63:2889-2934.

47. Lloyd-Jones DM, Morris PB, Ballantyne CM, et al. 2016 ACC expert consensus decision pathway on the role of non-statin therapies for LDL-cholesterol lowering in the management of atherosclerotic cardiovascular disease risk. J Am Coll Cardiol. 2016;68:92-125.

48. Landmesser U, Chapman MJ, Farnier M, et al. European Society of Cardiology/European Atherosclerosis Society Task Force consensus statement on proprotein convertase subtilisin/kexin type 9 inhibitors: practical guidance for use in patients at very high cardiovascular risk. Eur Heart J. Epub 2016 Oct 27

49. Banach M, Rizzo M, Toth PP, et al. Statin intolerance - an attempt at a unified definition: position paper from an international lipid expert panel. Arch Med Sci. 2015;11:1-23.

50. Kazi DS, Moran AE, Coxson PG, et al. Cost-effectiveness of PCSK9 inhibitor therapy in patients with heterozygous familial hypercholesterolemia or atherosclerotic cardiovascular disease. JAMA. 2016;316: 743-753.

51. Baum SJ, Toth P, Underberg JA, Jellinger P, Ross J, Wilemon K. PCSK9 inhibitor access barriers - issues and recommendations: improving the access process for patients, clinicians and payers. Clin Cardiol. 2017;40: 243-254.

52. Roth EM, Bujas-Bobanovic M, Louie MJ, Cariou B. Patient and physician perspectives on mode of administration of the PCSK9 monoclona antibody alirocumab, an injectable medication to lower LDL-C levels. Clin Ther. 2015;37:1945-1954.

53. Dent R, Joshi R, Stephen Djedjos C, et al. Evolocumab lowers LDL-C safely and effectively when self-administered in the at-home setting. Springerplus. 2016;5:300.

\section{Publish your work in this journal}

Drug Design, Development and Therapy is an international, peerreviewed open-access journal that spans the spectrum of drug design and development through to clinical applications. Clinical outcomes, patient safety, and programs for the development and effective, safe, and sustained use of medicines are the features of the journal, which

\section{Dovepress}

has also been accepted for indexing on PubMed Central. The manuscript management system is completely online and includes a very quick and fair peer-review system, which is all easy to use. Visit http://www.dovepress.com/testimonials.php to read real quotes from published authors. 